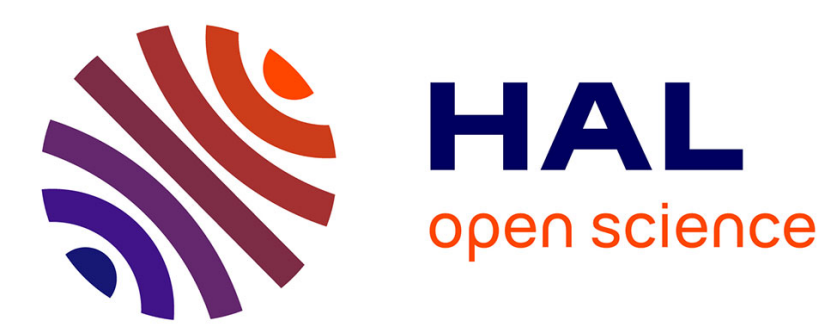

\title{
Description par l'opérateur densité de la réponse optique d'un état excitonique d'une assemblée de doubles chaines moléculaires orientées dans une matrice non résonnante
}

\author{
C. Aslangul, P. Kottis
}

\section{- To cite this version:}

C. Aslangul, P. Kottis. Description par l'opérateur densité de la réponse optique d'un état excitonique d'une assemblée de doubles chaines moléculaires orientées dans une matrice non résonnante. Journal de Physique Lettres, 1977, 38 (20), pp.413-416. 10.1051/jphyslet:019770038020041300 . jpa-00231409

HAL Id: jpa-00231409

https://hal.science/jpa-00231409

Submitted on 1 Jan 1977

HAL is a multi-disciplinary open access archive for the deposit and dissemination of scientific research documents, whether they are published or not. The documents may come from teaching and research institutions in France or abroad, or from public or private research centers.
L'archive ouverte pluridisciplinaire HAL, est destinée au dépôt et à la diffusion de documents scientifiques de niveau recherche, publiés ou non, émanant des établissements d'enseignement et de recherche français ou étrangers, des laboratoires publics ou privés. 


\title{
DESCRIPTION PAR L'OPÉRATEUR DENSITÉ DE LA RÉPONSE OPTIQUE D'UN ÉTAT EXCITONIQUE D'UNE ASSEMBLÊE DE DOUBLES CHAINES MOLÉCULAIRES ORIENTÉES DANS UNE MATRICE NON RÉSONNANTE
}

\author{
C. ASLANGUL \\ Centre de Mécanique Ondulatoire Appliquée, 23, rue du Maroc, 75109 Paris, France \\ et \\ P. KOTTIS \\ Laboratoire d'Optique Moléculaire (E.R. 134), Université de Bordeaux I, \\ 351, cours de la Libération, 33405 Talence Cedex, France \\ (Reçu le 8 juillet 1977, révisé le 15 septembre 1977, accepté le 19 septembre 1977)

\begin{abstract}
Résumé. - On généralise les résultats obtenus précédemment au cas d'une double chaîne ; même pour les valeurs assez élevées du nombre de sites, $N$, les effets de dimension finie sont manifestes sur le spectre.
\end{abstract}

\begin{abstract}
We generalize our previous results to an ensemble of double finite chains ; even for large numbers of sites, $N$, the effects of the finite size of the aggregate are seen in the spectrum.
\end{abstract}

1. Introduction. - Nous proposons une extension du travail déjà publié [1] portant sur le spectre optique d'un agrégat moléculaire fini inséré dans une matrice cristalline isotopique non résonnante, support d'un champ de phonons susceptible d'interagir avec les excitations électroniques élémentaires de l'agrégat.

La dimension finie des chaînes spécifiquement considérées, et pour laquelle on ne peut plus faire appel aux conditions simplificatrices de Born-von Karman, joue un rôle important pour la comprehension d'un spectre expérimental. L'étude expérimentale de tels systèmes est généralisée depuis 10 ans pour mettre en évidence les propriétés des associations moléculaires dans leur manifestation spectroscopique (raies supplémentaires) et dynamique (largeurs de raies, mouvement de l'excitation). En premier lieu il existe un ensemble complexe de règles d'intensité et de sélection, fonction de la géométrie de l'agrégat et déjà notée par Hochstrasser et Whiteman [6] pour une chaîne à sites parallèles. On constate que, même pour une valeur élevée du nombre de sites, $N(\sim 50)$, ces règles se manifestent encore en provoquant des élargissements inhomogènes assymétriques. En second lieu, pour $N \leqslant 10$, une résonance donnée n'a pas une forme lorentzienne : la susceptibilité totale contient deux contributions, l'une lorentzienne, l'autre non définie positive qui renforce l'absorption au centre et la diminue dans les ailes : globalement, la raie paraît plus finie qu'une lorentzienne, sans que son profil ait un lien quelconque avec une distribution gaussienne.

Cette note fait suite aux publications [1, 2, 4] en les généralisant au cas d'un agrégat formé de deux chaînes simples $\mathbf{A}$ et $\mathbf{B}$ en interaction limitée aux plus proches voisins et contenant chacune $N$ sites.

Soit $\hbar \Delta \omega$ l'interaction à l'intérieur d'une chaîne, et $\hbar \Delta \omega^{\prime}$ le couplage entre les deux chaînes ; le hamiltonien statique $\hat{H}_{0}$ s'écrit alors :

$$
\begin{aligned}
\hat{H}_{0}=\hbar \sum_{I=\mathrm{A}, \mathrm{B}}\left\{\omega_{0} \sum_{p=1}^{N}\left|M_{p}^{I}\right\rangle\left\langle M_{p}^{I}\left|+\Delta \omega \sum_{p=1}^{N-1}\right| M_{p}^{I}\right\rangle\left\langle M_{p+1}^{I}\right|\right. & \left.+\left|M_{p+1}^{I}\right\rangle\left\langle M_{p}^{I}\right|\right\}+ \\
& +\hbar \sum_{p=1}^{N} \Delta \omega^{\prime}\left(\left|M_{p}^{\mathrm{A}}\right\rangle\left\langle M_{p}^{\mathrm{B}}|+| M_{p}^{\mathrm{B}}\right\rangle\left\langle M_{p}^{\mathrm{A}}\right|\right) .
\end{aligned}
$$


Dans cette expression, $\left|M_{p}^{I}\right\rangle$ représente l'état d'ordre zéro monoexcité antisymétrisé construit sur le $p$-ième site de la chaîne $I(I=\mathrm{A}, \mathrm{B})$.

L'interaction exciton-phonon est décrite suivant le modèle de Haken et Strobl [3] ; conformément aux notations déjà introduites $[1,2,4], \gamma_{0}$ et $\gamma_{1}$ sont les fluctuations quadratiques moyennes locales et non locales à l'intérieur d'une chaîne : maintenant, $\gamma_{2}$ représente la fluctuation de l'élément de matrice dont la moyenne est $\Delta \omega^{\prime}$. L'équation d'évolution est donnée en (3.4) dans l'article [4] et la susceptibilité optique, $\chi(\omega)$, est calculée par la formule (4.5) de ce même article.

La détermination exacte de $\chi(\omega)$ est rendue possible par les propriétés remarquables de l'opérateur de relaxation, $\hat{\Gamma}$, qui trouvent leur généralisation ici.

Les vecteurs propres de $\hat{H}_{0}$ sont les $\left|A_{k b}\right\rangle$ tels que :

$$
\begin{gathered}
\hat{H}_{0}\left|A_{k b}\right\rangle=\hbar\left(\omega_{0}+2 \Delta \omega \cos k \theta+b \Delta \omega^{\prime}\right)\left|A_{k b}\right\rangle \equiv \hbar \omega_{k b}\left|A_{k b}\right\rangle \quad\left(\theta=\frac{\pi}{N+1}\right) \\
\left|A_{k b}\right\rangle=\left(\frac{1}{N+1}\right)^{1 / 2} \sum_{p=1}^{N} \sin k p \theta\left(\left|M_{p}^{\mathrm{A}}\right\rangle+b\left|M_{p}^{\mathrm{B}}\right\rangle\right) \quad(1 \leqslant k \leqslant N, b= \pm 1)
\end{gathered}
$$

et conduisent à définir les opérateurs $\hat{a}_{k b}^{+}$par la relation :

$$
\hat{a}_{k b}^{+}=\left|A_{k b}\right\rangle\langle 0| \quad(|0\rangle=\text { état fondamental }) .
$$

Après un calcul simple, on obtient :

$$
\left(a_{k b}^{+}|\hat{H}| a_{k^{\prime} b^{\prime}}^{+}\right)=\hbar\left\{\omega_{k b}-i\left(\gamma_{0}+2 \gamma_{1}+\gamma_{2}\right)\right\} \delta_{k k^{\prime}} \delta_{b b^{\prime}}+i \hbar \gamma_{1}\left(a_{k b}^{+}|\hat{P}| a_{k^{\prime} b^{\prime}}^{+}\right) .
$$

L'apparition de l'opérateur $\hat{P}$ est une conséquence directe de la dimension finie de l'agrégat; il est en fait la somme de quatre projecteurs orthogonaux et normalisés ; ceci permet de resommer analytiquement le développement de perturbation par rapport à $\hat{P}$ pour arriver aux résultats suivants [5] :

$$
\begin{aligned}
& \chi^{\prime \prime}(\omega)=\chi_{+1}^{\prime \prime}(\omega)+\chi_{-1}^{\prime \prime}(\omega) \quad\left(\chi^{\prime \prime}=-\operatorname{Im} \chi(\omega)\right) \\
& \chi_{b}^{\prime \prime}(\omega)=\frac{2 r d^{2} \omega_{0}^{2}}{\hbar N(N+1)}\left\{\sum_{k=1}^{N} \frac{T_{k b}^{2}}{4} \frac{\Gamma_{\mathrm{d} c}}{\left(\omega-\omega_{k b}\right)^{2}+\Gamma_{\mathrm{dc}}^{2}}+\right. \\
& \quad+\frac{4 \gamma_{1}}{N+1} \operatorname{Im}\left[\sum_{c=0,1} F_{c b}(\omega)\left(\left[\frac{\left[{ }^{N+c}\right.}{2} \sum_{k \geqslant 1} \frac{T_{k b}}{2} \frac{\sin \kappa \theta}{\left(\omega-\omega_{k b}\right)+i \Gamma_{\mathrm{dc}}}\right)\right]\right\}\left(\Gamma_{\mathrm{d} c}=\gamma_{0}+2 \gamma_{1}+\gamma_{2}\right) .
\end{aligned}
$$

Dans cette expression, $[x]$ désigne la partie entière du réel $x ; \kappa$ est un entier compris entre 1 et $N$, pair si $c=0$, impair si $c=1 ; F_{c b}$ est une fonction à valeurs complexes s'exprimant à l'aide des polynômes de Gegenbauer; $T_{k b}$ est le facteur géométrique introduisant la configuration de l'agrégat :

$$
T_{k}^{I}=\sum_{p=1}^{N} \mathbf{u} \cdot \mathbf{m}_{p}^{I} \sin k p \theta, \quad T_{k b}=T_{k}^{\mathrm{A}}+b T_{k}^{\mathrm{B}}
$$

$\mathbf{m}_{p}^{I}$ étant le vecteur unitaire porté $\operatorname{par}\left\langle 0\left|\sum_{i} e \mathbf{r}_{i}\right| M_{p}^{I}\right\rangle$ et $\mathbf{u}$ la polarisation du champ extérieur. On note que chaque $T_{k}^{I}$ est le facteur géométrique d'une simple chaîne; les règles de sélection globales seront donc en quelque sorte le produit de celles du dimère par celles d'une chaîne simple.
On voit que le spectre sera la superposition de deux séries de résonances $(b= \pm 1)$; chaque série est centrée en $\omega_{0} \pm \Delta \omega^{\prime}$ et peut contenir $N$ raies au plus. Chaque résonance présente un écart à la forme lorentzienne fourni par la contribution proportionnelle à $4 \gamma_{1} /(N+1)$, elle-même résultant de la longueur finie de l'agrégat. Ces effets de bords deviennent tout à fait négligeables pour $N \gtrsim 20$; l'aspect fini du système continue toutefois de se manifester pour les valeurs élevées du nombre de sites $(N \sim 50)$, par de forts élargissements inhomogènes aux extrémités du spectre.

Les $T_{k b}$ définissent les intensités relatives des différentes résonances et n'ont une expression intéressante que dans la mesure où $N$ est fini ; comme précédemment, nous avons considéré les trois types suivants de configuration : 
2. Chaînes parallèles. - C'est le cas où $\left(\mathbf{u}, \mathbf{m}_{p}^{I}\right)=\psi^{I}$, indice $k$ est : angle constant indépendant de $p$. On trouve alors :

$$
T_{k b}=\frac{1-(-1)^{k}}{2} \operatorname{cotg}^{2} k \frac{\theta}{2}\left(\cos \psi^{\mathrm{A}}+b \cos \psi^{\mathrm{B}}\right) \text {. }
$$

Pour chaque série, on a donc une décroissance très rapide de l'intensité lorsque $k$ augmente, les raies paires étant strictement interdites: de surcroît, le rapport des intensités des deux raies ayant le même

$$
\left(\frac{\cos \psi^{\mathrm{A}}+\cos \psi^{\mathrm{B}}}{\cos \psi^{\mathrm{A}}-\cos \psi^{\mathrm{B}}}\right)^{2}
$$

qui coïncide avec le rapport des deux résonances dimériques $[2,4]$. La figure 1 donne un exemple de spectre lorsque les interactions interchaînes sont petites devant les interactions intrachaînes $\left(\left|\Delta \omega^{\prime}\right| \ll|\Delta \omega|\right)$.

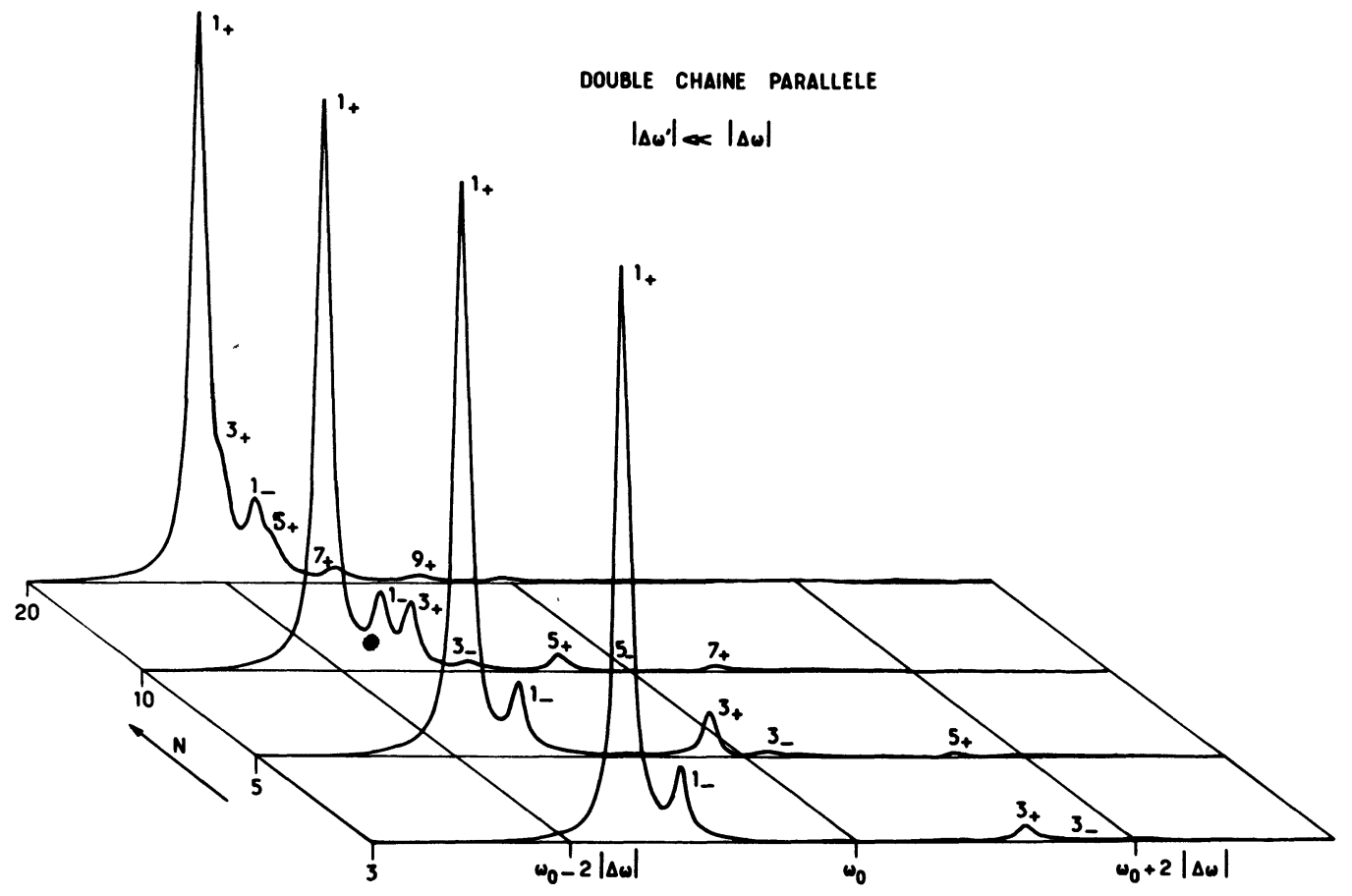

Fig. 1. - Spectre total d'absorption d'une double chaîne formée de deux agrégats parallèles. [Absorption spectrum of a double chain, formed by two parallel aggregates.]
3. Chaînes alternées. - On a ici, par définition
$\left(\mathbf{u}, \mathbf{m}_{2 p+1}^{I}\right)=\psi^{\prime I}$
$\left(\mathbf{u}, \mathbf{m}_{2 p}^{I}\right)=\psi^{\prime \prime I}$

Un calcul simple donne alors :

$$
\begin{aligned}
T_{k}^{I}=\frac{1-(-1)^{N}}{2} \frac{1-(-1)^{k}}{2} \frac{\cos \psi^{\prime I}+\cos k \theta \cos \psi^{\prime \prime I}}{\sin k \theta}+ \\
\quad+\frac{1+(-1)^{N}}{2} \frac{1-(-1)^{k} \cos k \theta}{2} \frac{\cos \psi^{\prime I}-(-1)^{k} \cos \psi^{\prime \prime I}}{\sin k \theta}
\end{aligned}
$$

La figure 2 donne un exemple de tel spectre pour $|\Delta \omega| \ll\left|\Delta \omega^{\prime}\right|$ : on note dans ce cas l'existence d'un doublet fortement structuré par les différentes valeurs de l'indice $k$.

4. Chaînes en hélice. - Il n'existe de règles de sélection simples que si le quantum de rotation, $\Delta \psi$, est un multiple entier de l'angle $\theta$, soit $\Delta \psi=k_{0} \theta$ : alors, toutes les raies de même parité que $k_{0}$ sont interdites, à l'exception toutefois de la résonance $k_{0}$ elle-même, dont l'intensité relative par rapport aux raies permises peut être faible ou forte selon les cas. $\mathrm{La}$ figure 3 présente le résultat du calcul exact avec $\Delta \psi^{\mathrm{A}}=\Delta \psi^{\mathrm{B}}=\theta$, lorsque, de surcroît, les interactions sont supposées $\left(|\Delta \omega| \gg\left|\Delta \omega^{\prime}\right|\right)$.

En conclusion, on voit que la dimension finie de l'agrégat joue un rôle essentiel pour la forme du spectre optique. Pour les petites valeurs de $N(\lesssim 10)$ une résonance donnée présente un écart à la forme lorentzienne : plus intense au centre, elle est plus faible dans les ailes, sans présenter aucun rapport avec le profil gaussien. Pour les valeurs élevées de $N(\sim 50)$, cette propriété peut être ignorée, mais alors la taille finie se manifeste par de forts élargissements inhomogènes observés parce que, au contraire d'une chaîne infinie, il y $a$ ici un très grand nombre de raies permises : la densité d'états étant plus forte aux extrémités du spectre, il est normal de trouver à ces endroits les élargissements les plus importants, dont il est facile de montrer, par un passage à la limite, qu'ils disparaissent si $N \rightarrow \infty$. 


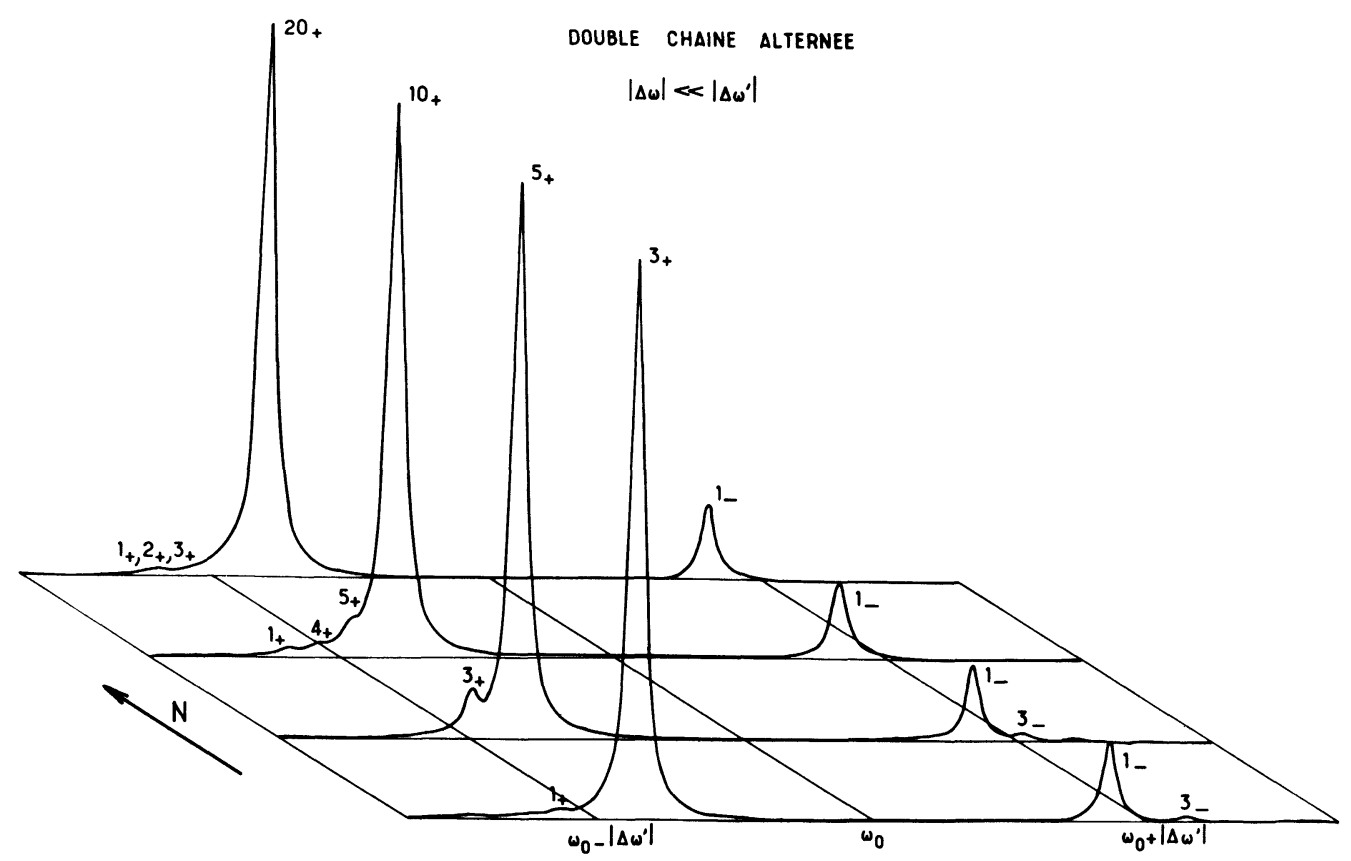

Fig. 2. - Spectre total d'absorption d'une double chaîne, formée de deux agrégats alternés. [Absorption spectrum of a double chain formed by two alternate aggregates.]

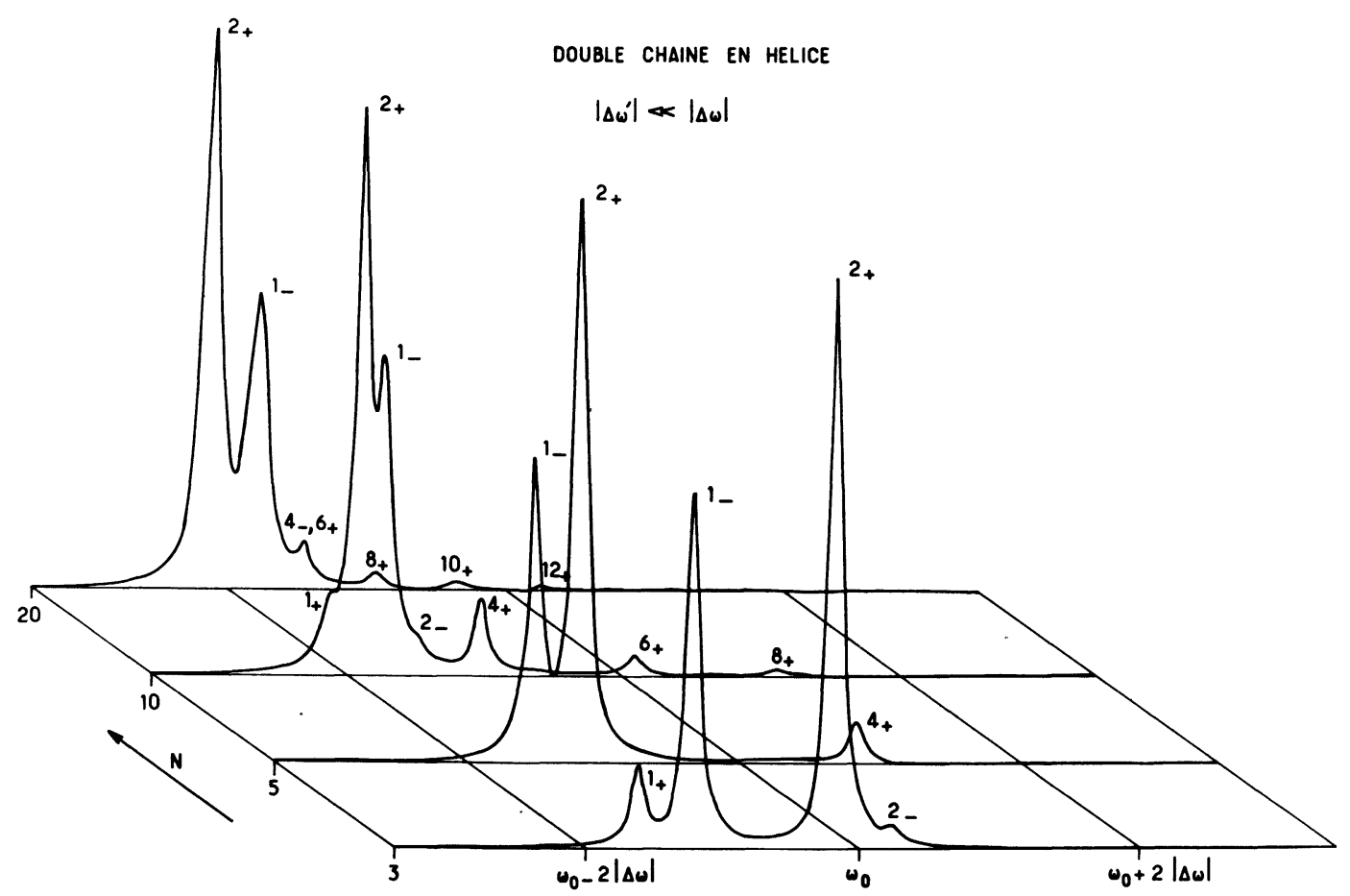

FIG. 3. - Spectre total d'absorption d'une double chaîne formée de deux agrégats en hélice (la polarisation de l'excitation est perpendiculaire à l'axe de l'hélice).

[Absorption spectrum of a double chain formed by two helical aggregates (the excitation polarization is perpendicular to helix axis).]

\section{Bibliographie}

[1] Aslangul, Cl., Kottis, Ph., Phys. Rev. B 13 (1976) 5544.

[2] Aslangul, Cl., Kottis, Ph., C. R. Hebd. Séan. Acad. Sci. B 279 (1974) 523.

[3] Haken, H., Strobl, G., dans The Triplet State, édité par A. B. Zahlan (Cambridge University Press) 1968.
[4] Aslangul, Cl., Kottis, Ph., Phys. Rev. B 10 (1974) 4364.

[5] Aslangul, Cl., Thèse d'Etat, Paris 6, Avril 1977.

[6] Hochstrasser, R. M., Whiteman, J. D., J. Chem. Phys. 56 (1972) 5945 ;

Whiteman, J. D., Thèse (University of Pennsylvania, 1971). 